Domingo-Coscollola, M.; Onsès-Segarra, J. y Sancho-Gil, J.M. (2018). La cultura DIY en educación primaria. Aprendizaje transdisciplinar, colaborativo y compartido en DIYLabHub. Revista de Investigación Educativa, 36(2), 491-508.

DOI: http://dx.doi.org/10.6018/rie.36.2.304421

\title{
La cultura DIY en educación primaria. Aprendizaje transdisciplinar, colaborativo y compartido en DIYLabHub
}

\section{DIY culture in primary school.Transdisciplinar and collaborative learning shared in DIYLabHub}

\author{
Maria Domingo-Coscollola*, Judit Onsès-Segarra ${ }^{* *}$ y Juana M. Sancho-Gil ${ }^{* *}$ \\ * Facultad de Educación. Universitat Internacional de Catalunya \\ ${ }^{*}$ Facultad de Educación. Universitat de Barcelona
}

\begin{abstract}
Resumen
Este artículo da cuenta del proyecto europeo DIYLab y de su implementación en una escuela de educación primaria de España. El proyecto se desarrolló en centros de educación primaria, secundaria y superior de España, Finlandia y Chequia. El foco era incorporar, en las instituciones implicadas, modalidades de aprender relacionadas con la cultura Do it Yourself (DIY). Siguiendo una metodología basada en los principios de la investigación-acción colaborativa, organizamos grupos de discusión con familias, profesorado y alumnado. También, definimos su implementación partiendo de las características de cada centro y de las acciones formativas en el profesorado. Durante la formación, se planteó que los estudiantes realizaran de forma colaborativa una producción audiovisual sobre sus aprendizajes y los procesos que los habian propiciado. A su vez, se decidió en qué proyecto de indagación del centro se implementaría DIYLab para desarrollar características de la cultura DIY tales como la creatividad, la colaboración, la autorregulación, la autoría, la compartición y el uso de tecnologías. Simultáneamente, proporcionamos la plataforma abierta DIYLabHub creada para este proyecto donde se podían
\end{abstract}

Correspondencia: María Domingo-Coscollola, mdomingoc@uic.es, Universitat Internacional de Catalunya (UIC), Facultad de Educación, España. 
compartir las producciones audiovisuales de los estudiantes. Durante la implementación, los investigadores realizamos observaciones, grabaciones y notas de campo. A partir de estas fuentes, centramos los resultados y conclusiones en las producciones audiovisuales que estudiantes de educación primaria de España crearon colaborativamente y compartieron en el Hub así como en las fortalezas y debilidades del proyecto en torno a las relaciones pedagógicas, la competencia digital y la sostenibilidad del proyecto.

Palabras clave: aprendizaje; investigación-acción colaborativa; educación primaria; producción audiovisual.

\section{Abstract}

This paper accounts for the DIYLab European project and its implementation in a Spanish primary school. The project was developed in primary, secondary and higher education institutions in Spain, Finland and the Czech Republic. The focus was to incorporate, in the organizations involved, learning modalities related to Do it Yourself(DIY) culture. Following a methodology based on the principles of the collaborative action-research, we planned discussion groups with families, teachers and students. In addition, we defined its implementation starting from the characteristics of each institution and the professional development of teachers. During this action, it was decided that students collaboratively performed an audio-visual production about their learning and the processes that had propitiated it. It was also decided in which research-based project DIYLab would be implemented, to develop features of DIY culture such as creativity, collaboration, self-regulation, authorship, sharing and use of technologies. Simultaneously, the open platform DIYLabHub was also provided so that students' audiovisual productions could be shared. Throughout this implementation, researchers made observations, recordings and took field notes. From these sources, we focused the results and conclusions on audio-visual productions that primary school students from Spain created collaboratively and shared in the Hub, as well as on the strengths and weaknesses regarding pedagogical relations, digital competences and sustainability of the project.

Keywords: learning; collaborative action-research; primary school; audiovisual production.

\section{Introducción}

Los medios digitales y el uso de Internet forman parte de las prácticas de niños y jóvenes. Esta realidad está contribuyendo a cambiar sus dinámicas en relación con el aprendizaje, la socialización y el acceso a la información. En este contexto, destacamos las prácticas que fomentan las "redes sociales y la creación de contenido" (Richard \& Kafai, 2016, p. 1478) y también las prácticas de aprender en colaboración afrontando retos relacionados, entre otros, con proyectos musicales (Fields, Vasudevan, \& Kafai, 2015, p. 613), medios audiovisuales (Jenson, Dahya, \& Fisher, 2014, p. 224), artísticos (Peppler, 2013, p. 6) y diseño textil digital (Kafai \& Peppler, 2014, p. 179). Todas estas prácticas tienen en común que en ellas "existe una cultura de participación" (Jenkins, Purushotma, Weigel, Clinton, \& Robison, 2009, p. 3). A título de ejemplo, señalamos las prácticas que potencian "la adquisición de habilidades y conocimientos individuales añadiendo valor a una comunidad al compartir su propio conocimiento" (Ito et al., 2013, 
p. 7) o las que tienen lugar en "entornos de aprendizaje conectados que reúnen jóvenes y/o adultos en actividades conjuntas con propósito compartido" (Ito et al., 2013, p. 75).

De este modo, sobre todo fuera de los centros educativos, existen prácticas que están configurando aprendizaje y socialización, a la vez que van desarrollando otras maneras de aprender. Estos nuevos contextos generan nuevos interrogantes, desafíos y posibilidades para los educadores, las familias y los responsables de las políticas educativas. Ante este contexto, distintos autores empiezan a reconocer y a plantearse cómo y dónde aprenden los niños y los jóvenes (Bell, Tzou, Bricker, \& Baines, 2013; Buckingham, 2007; Carey, 2014; Erstad, Gile, Sefton-Green, \& Arnseth, 2016), para intentar explorar nuevas culturas de aprendizaje (Erstad et al., 2016; Hernández-Hernández, 2017; Livingstone \& Sefton-Green, 2016; Thomas \& Brown, 2011). Esta realidad, nos lleva a la necesidad de explorar unas nuevas culturas que permiten contextualizar y expandir modos de aprender que amplían relaciones, referentes y planteamientos educativos.

En este nuevo contexto, ubicamos el proyecto europeo en el que se basa este artículo: DIYLab - Do It Yourself in Education: Expanding Digital Competence to Foster Student Agency and Collaborative Learning. El proyecto se desarrolló entre 2014 y 2016 implementándose en centros educativos de primaria, secundaria y educación superior de España, Finlandia y Chequia. Se trata de una iniciativa que conecta con los argumentos planteados previamente y con distintos estudios sobre las realidades y retos del aprendizaje de los jóvenes en la era digital (Ashworth, 2016; Boyd, 2014; Carey, 2014; Knobel \& Lankshear, 2010; Palaigeorgiou \& Grammatikopoulou, 2016; Pérez-Escoda, Castro-Zubizarreta, \& Fandos, 2016; Sharpe, Beethan, \& De Freitas, 2010; Shirky, 2010).

El foco del proyecto DIYLab era incorporar, en las instituciones implicadas, modalidades de aprender relacionadas con la cultura Do it Yourself (DIY). A modo de ejemplo señalamos que "en las redes DIY, los jóvenes de forma voluntaria dedican tiempo a un aprendizaje intenso y abordando prácticas de alto nivel técnico" (Kafai \& Peppler, 2011, p. 89). A partir de este foco, nuestro reto era introducir (en cada institución implicada) modos de acción y prácticas de enseñanza y aprendizaje surgidas en contextos informales. Así, pretendíamos contextualizar el aprendizaje generando nuevos interrogantes, posicionamientos y planteamientos en las prácticas de los centros implicados.

La finalidad principal del proyecto DIYLab era fomentar el aprendizaje a lo largo y ancho de la vida (Banks et al., 2007) mediante el desarrollo de la competencia digital crítica (Ala-Mutka, 2009; Gutiérrez \& Tyner, 2013), la capacidad de acción y la creatividad. Además, pretendíamos avivar el compromiso de los estudiantes proponiendo experiencias de aprendizaje colaborativas y significativas que pudieran ser sostenibles y expandibles al finalizar el proyecto. De ahí que los principales objetivos de este proyecto eran:

1. Establecer espacios flexibles (denominados DIYLabs) para desarrollar actividades curriculares transversales donde los estudiantes pudieran realizar proyectos de indagación de forma colaborativa conectando diferentes materias con sus intereses.

2. Construir a través de un proceso de formación colaborativa (entre investigadores y profesorado) un enfoque conceptual que permitiera a los estudiantes producir y compartir conocimiento, y autorregular su aprendizaje, contemplando la competencia digital y la creatividad. 
3. Definir y evaluar el diseño e implementación de los DIYLabs mediante un proceso de investigación en la acción colaborativa.

4. Desarrollar la plataforma digital DIYLabHub (http://hub.diylab.eu) para compartir y crear comunidades de aprendizaje donde el alumnado pudiera depositar las producciones audiovisuales creadas de forma colaborativa sobre sus aprendizajes y los procesos que los han propiciado.

Así, planteamos el proceso de repensar la educación incidiendo en la realización de transformaciones y mejoras de la práctica educativa de los centros implicados en el proyecto. Destacamos qué acciones significativas facilitaron este proceso:

- Revisar y modificar el currículo para poder asumir problemáticas no visibles en la enseñanza tradicional y generar otras posibilidades de aprender sobre lo que se desconoce (Atkinson, 2011).

- Entender la acción de enseñar y aprender como un acto basado en las emociones e interacciones humanas (Maturana, 1990), y en la capacidad para ponerse en la posición del otro, de ayudar y de impulsar el "aprender de los otros y con los otros" (Domingo-Coscollola, Sánchez-Valero, \& Sancho-Gil, 2014, p. 163).

- Contribuir a encontrar espacios de aprendizaje para que el alumnado pueda profundizar las ideas conocidas y descubrir y hacer cosas que no sabía ni se esperaba (Atkinson, 2011). En este caso, considerando que no todos los alumnos llegarían al mismo lugar ni de igual forma y, a la vez, contemplando la posibilidad de que pudieran usar distintas vías y distintos lenguajes para dar cuenta de sus procesos de aprendizaje.

Finalmente, en este artículo, además de la visión de conjunto del proyecto europeo DIYLab, damos cuenta de su implementación en la Escola Virolai. Un centro concertado situado en el barrio del Carmel de Barcelona que abarca desde primer ciclo de Educación Infantil hasta Bachillerato y que decidió aplicar este proyecto en sus dos líneas de quinto curso de educación primaria. Este centro se define como una escuela viva consciente de que la sociedad donde nos encontramos está sujeta a cambios continuos (Escola Virolai, s.f.). La escuela se interesó en participar en este proyecto por su planteamiento y por su posible contribución en avanzar hacia una educación centrada en los alumnos y el mundo en el que viven, y que facilite el aprendizaje vivencial, significativo y continuado. Una de las singularidades de esta escuela es su participación en proyectos educativos con otros centros y en investigaciones planteadas desde la universidad (Domingo-Coscollola, Sánchez-Valero, \& Sancho-Gil, 2014; HernándezHernández \& Domingo-Coscollola, 2017).

\section{Método}

El proyecto DIYLab comenzó con la preparación de su propuesta y su presentación a la Comisión Europea. Esta propuesta fue elaborada por las instituciones de tres países (ver tabla 1). El planteamiento del proyecto, sus informes técnicos y su producción científica está disponible con acceso abierto en http://diylab.eu/. 
Tabla 1

Centros educativos implicados en el proyecto

\begin{tabular}{ccc}
\hline \multicolumn{1}{c}{ País } & Institución & Nivel educativo \\
\hline \multirow{2}{*}{ España } & Escola Virolai (Barcelona) & Primaria y secundaria \\
& Universitat de Barcelona & Universidad \\
Finlandia & Oulu University Teacher Training School & Primaria y secundaria \\
& University of Oulu & Universidad \\
Chequia & ZŠ Korunovační (Praga) & Primaria y secundaria \\
& Charles University (Praga) & Universidad \\
\hline
\end{tabular}

Este proyecto se desarrolló con el fin primordial de transformar las prácticas de enseñanza y aprendizaje y de consolidar los cambios una vez finalizado el proyecto. Para ello, seguimos una metodología basada en los principios de la investigaciónacción colaborativa:

Un proceso participativo y democrático orientado a desarrollar el conocimiento práctico mediante la búsqueda de objetivos humanos valiosos, basado en una cosmovisión participativa que creemos que está emergiendo en este momento histórico. Busca reunir la acción y la reflexión, la teoría y la práctica, en la participación con otros, en la búsqueda de soluciones prácticas a cuestiones preocupantes para la gente y, más en general, el florecimiento de las personas individuales y sus comunidades (Reason \& Bradbury, 2001, p. 1).

La tabla 2 ofrece una visión de conjunto de la implementación del proyecto en los distintos niveles de las instituciones participantes destacando qué número de estudiantes, docentes y asignaturas estaban implicadas en las producciones audiovisuales realizadas por el alumnado de los tres países.

Tabla 2

Visión general del Proyecto DIYLab

\begin{tabular}{lccccc}
\hline País & Nivel educativo & Estudiantes & Docentes & Asignaturas & $\begin{array}{c}\text { Producciones } \\
\text { DIYLabHub }\end{array}$ \\
\hline \multirow{2}{*}{ España } & Primaria y secundaria & 95 & 15 & 9 & 32 \\
\multirow{2}{*}{ Finlandia } & Universidad & 471 & 20 & 11 & 76 \\
& Primaria y secundaria & 114 & 14 & 18 & 56 \\
& Primaria y secundaria & 269 & 7 & 13 & 20 \\
& Universidad & 242 & 8 & 17 & 33 \\
\hline
\end{tabular}


Los métodos utilizados a lo largo del proyecto fueron:

- Análisis crítico de documentos (Bowen, 2009).

- Grupos de discusión (Barbour \& Kitzinger, 1999).

- Práctica basada en la investigación (Furlong \& Oancea, 2008).

- Observación, notas de campo y documentación visual (Dussel \& Gutiérrez, 2006; Van Maanen, 2011).

Los grupos de discusión se realizaron al inicio y final del proyecto implicando a docentes, familiares y estudiantes de distintos niveles educativos y países (ver tabla 3).

Tabla 3

Número total de participantes en los grupos de discusión, por país y nivel de enseñanza

\begin{tabular}{|c|c|c|c|c|}
\hline País & Nivel educativo & Docentes & Familiares & Estudiantes \\
\hline \multirow{4}{*}{ España } & \multirow{2}{*}{$\begin{array}{l}\text { Primaria y } \\
\text { secundaria }\end{array}$} & 9 Primaria & 9 Primaria & 10 Primaria \\
\hline & & 10 Secundaria & 11 Secundaria & 11 Secundaria \\
\hline & Universidad & 12 de 5 dptos. & --- & 14 de 4 grados \\
\hline & \multirow{3}{*}{$\begin{array}{l}\text { Primaria y } \\
\text { secundaria }\end{array}$} & 8 Combinados & 10 Combinados & 8 Combinados \\
\hline \multirow[t]{2}{*}{ Finlandia } & & 6 Primaria & 6 Primaria & 34 Primaria \\
\hline & & 6 Secundaria & 4 Secundaria & 6 Secundaria \\
\hline \multirow{3}{*}{ Chequia } & \multirow{2}{*}{$\begin{array}{l}\text { Primaria y } \\
\text { secundaria }\end{array}$} & 12 Primaria & 8 Primaria & 15 Primaria \\
\hline & & 11 Secundaria & 9 Secundaria & 16 Secundaria \\
\hline & Universidad & $\begin{array}{c}7 \text { de asignaturas } \\
\text { diferentes }\end{array}$ & --- & 49 de máster \\
\hline TOTAL & & 81 & 57 & 163 \\
\hline
\end{tabular}

Este proyecto se inició en 2014 (fase 1). En esta fase, se preparó la especificación e implementación del proyecto partiendo de las características de cada centro y de la formación de su profesorado. En 2015 (fase 2), se implementaron los DIYLabs en las instituciones implicadas y se desarrolló el DIYLabHub. En 2016 (fase 3), se analizaron las implementaciones y se realizó la evaluación socioeconómica. Durante todo el proceso (figura 1), realizamos observaciones, grabaciones y notas de campo existiendo un seguimiento externo de garantía de calidad por ser un proyecto europeo.

Durante el diseño y la implementación del proyecto, se analizó y dialogó sobre la práctica educativa en el aula llegando a consensos en cada centro educativo. A partir del análisis de las fuentes ya señaladas, destacamos los consensos más significativos:

- Durante la revisión del rol del docente, se apuesta por un docente con alto nivel de implicación, interés, y motivación y que favorece la conexión entre el dentro y el fuera del centro educativo. 
Fase 1. Diseño

Construir el DIYLab a partir de la experiencia y saberes de los participantes

Formación para diseñar la implementación del DIYLab en los centros educativos
Fase 2. Implementación

Los DIYLabs en acción

Lanzamiento de DIYLabHub
Fase 3. Evaluación y mejoras

Mejoras para el DIYLab a partir de la experiencia vivida

Evaluación socio-económica

Figura 1. Fases del proceso de investigación-acción colaborativa del proyecto DIYLab.

- Se reafirma la idea de aprender mediante proyectos basados en la indagación, propiciando la transdisciplinariedad, la pluralidad y la flexibilidad, con el fin de ampliar los límites del conocimiento conectando el dentro y fuera de la escuela.

- Se implica el docente y/o el investigador acompañando al alumnado para proponer, cuestionar y construir el desarrollo del proyecto de indagación de los estudiantes.

- Se establecen espacios dentro de las asignaturas de cada uno de los docentes implicados para conseguir el aprendizaje transdisciplinar y colaborativo del proyecto.

- Se crean espacios no regulados por el tiempo donde el estudiante pueda ir creando, a su ritmo, material de aprendizaje transdisciplinar en contextos estimulantes.

También, destacamos que el profesorado centró su atención en aspectos relacionados con los procesos de reflexión sobre el aprendizaje y la importancia de compartir el conocimiento adquirido con personas de todos los países a través de la creación de una plataforma digital abierta (DIYLabHub).

Seguidamente, especificamos el contenido significativo de los momentos o fases de la investigación-acción colaborativa.

\section{Fases de la investigación-acción colaborativa}

Durante 2014 (fase 1), se realizaron diferentes acciones para involucrar a todas las instituciones implicadas en el proyecto y preparar la definición e implementación de los DIYLabs, partiendo de sus características y culturas para poder contribuir al desarrollo de su capacidad de liderazgo educativo (Domingo-Coscollola, Arrazola-Carballo, \& Sancho-Gil, 2016). Para ello:

- Se analizaron los currículos oficiales y los planes de estudios de los centros implicados teniendo en cuenta la cultura DIY. Esto permitió identificar (en cada uno de ellos) las áreas curriculares, los espacios y momentos más convenientes 
para introducir experiencias de aprendizaje vinculadas a la cultura DIY y a la realización de las producciones audiovisuales que se podían compartir de forma abierta en el DIYLabHub.

- A través de grupos de discusión, se exploraron las opiniones y los posicionamientos sobre la posible implementación del proyecto, por parte del alumnado, del profesorado y de las familias. El contenido de los grupos de discusión iniciales se analizó a partir de distintos indicadores: aprendizaje autorregulado, aprendizaje autónomo, aprendizaje colaborativo, metarreflexión, transferencia de lo aprendido, evaluación del aprendizaje como actividad reflexiva y uso de recursos para el aprendizaje.

- Se realizó una acción formativa para todos los implicados en el proyecto. Durante esta formación, se procedió a definir, diseñar e imaginar posibles escenarios y propuestas de implementación de la cultura DIY en cada institución. En todos los casos, se decidió optar por la perspectiva de los proyectos basados en la indagación.

- Se plantearon preguntas orientativas para guiar las producciones audiovisuales colaborativas del alumnado sobre sus aprendizajes y los procesos que los propiciaron: qué hemos hecho, cómo lo hemos hecho, qué hemos aprendido y cómo lo hemos aprendido. A la vez, se propuso el desafío de promover seis características de la cultura DIY en los procesos de enseñanza y aprendizaje: creatividad, colaboración, autorregulación, autoría, compartición y uso de tecnologías.

Durante 2015 (fase 2), se implementaron los DIYLabs en cada institución participante según sus particularidades, pero contemplando puntos básicos y compartidos tales como:

- La colaboración como clave de la relación pedagógica. Esto implicó fomentar la creación de un sentimiento de grupo y de autorizarse desde la implicación, promoviendo el debate, la toma de decisiones y la acción conjunta.

- El papel de las tecnologías (no sólo digitales) como mediadoras de los aprendizajes y modos de representarlos.

- Las decisiones creativas desde la adaptación, la transformación y la experimentación.

- La realización de producciones audiovisuales sobre los aprendizajes de los estudiantes y los procesos que los habían propiciado.

- La consideración del rol de los estudiantes como prosumidores y autores de conocimiento (García-Galera \& Valdivia, 2014).

- La autorregulación relacionada con la capacidad de los implicados para dirigir su aprendizaje tomando consciencia y aumentando su autoconfianza (Zimmerman, 1989, p. 10).

En el ciclo de primaria de la Escola Virolai, la implementación del DIYLab se realizó aplicando la metodología de aprendizaje basado en problemas (García-Valcárcel \& Basilotta, 2017). Concretamente, la implementación en este centro educativo se llevó a término a través del proyecto de indagación Tivo Creativo, una propuesta transdisciplinar 
(incluyendo ciencias sociales, lengua catalana y lengua castellana) que proponía a los estudiantes diseñar una aplicación móvil para mejorar su vida cotidiana. Fruto de esta implementación, los 58 alumnos de quinto curso de educación primaria realizaron en grupo, con sus profesores, quince producciones audiovisuales sobre sus procesos de aprendizaje. Este alumnado se agrupó según sus intereses realizando y grabando sus producciones a partir de entrevistas entre pares. Para analizar las quince producciones audiovisuales y su proceso, dos investigadores realizamos observaciones en el aula y diarios de campo, así como entrevistas a los docentes y estudiantes implicados.

Durante 2016 (fase 3), se procedió al análisis de la implementación del proyecto como un todo, incluidas las producciones audiovisuales, para proponer elementos de mejora y afianzar los aspectos positivos del proyecto en la cultura docente. Para ello, realizamos dos acciones: 1) analizar los procesos y resultados de las experiencias de enseñanza y aprendizaje llevadas a cabo, y 2) organizar grupos de discusión para recoger las experiencias y valoraciones.

El análisis de los procesos y del contenido de los grupos de discusión finales se llevó a cabo a través de las siguientes dimensiones:

- Aprendizaje DIYLab: ambientes creados, relaciones con los currículos, diferencias con otros tipos de actividades donde la filosofía DIY no interviene, valoración del proyecto, reflexiones y sugerencias.

- Actitudes y roles: modo de relacionarse con el aprendizaje, existencia de cambios (motivación, desafección, desorientación, dudas, rol) con respecto a otros tipos de actividades donde la filosofía DIY no interviene.

- Competencia digital: habilidades digitales, descubrimientos y realidad tecnológica.

- Sostenibilidad del proyecto: continuidad y valoraciones (fortalezas, retos, debilidades y dificultades).

\section{Resultados}

En este apartado, recogemos los principales resultados fruto de la implementación del proyecto DIYLab en el ciclo superior de primaria de la Escola Virolai. Estos resultados están organizados en torno a la creación colaborativa de las producciones audiovisuales de los estudiantes, y las fortalezas y debilidades de la implementación del proyecto.

\section{Producciones audiovisuales: creación colaborativa compartida en DIYLabHub}

En primer lugar, presentamos los resultados significativos sobre la creación de las quince producciones audiovisuales transdisciplinares realizadas por estudiantes de quinto curso de educación primaria y que compartieron en DIYLabHub.

A modo de síntesis, la creación de estas producciones ha posibilitado:

- Reflejar otras nociones del estudiante, conociendo facetas, intereses y saberes de ellos que normalmente no mostraban, además de que cada uno fuera creando conocimiento progresivamente. 
- Desarrollar la competencia digital desde la finalidad principal de compartir aprendizajes de forma comprensible para una comunidad más allá del centro. En este caso, usando y relacionándose de forma diferente con las tecnologías digitales.

- Compartir sus aprendizajes con otros estudiantes, lo que significó prestar más atención a cómo ellos y sus compañeros aprendían y a qué aprendían a partir de un trabajo colaborativo sobre y desde el aprendizaje.

Referente al trabajo colaborativo realizado, se constata que ha fomentado:

- La redistribución de las jerarquías de saber o poder entre docentes y estudiantes y entre estudiantes.

- La reconsideración del rol del estudiante y del docente dentro del aula, y los nuevos modos de relación con los espacios y el personal del centro. En este caso, los estudiantes adoptaron los roles de aprendiz, enseñante y documentalista.

- El aumento del compromiso y la motivación de los estudiantes por su trabajo del que dependían los compañeros del grupo y que ubicarían, en forma de audiovisual, en una plataforma abierta.

- La necesidad de compartir puntos de vista o criterios diferentes, de consensuar opiniones y negociar actitudes incrementando el respeto hacia los compañeros.

A la vez, usar y disponer del DIYLabHub (para compartir las producciones) ha permitido a los estudiantes:

- Interesarse y aprender otra manera de compartir sus aprendizajes familiarizándose con un entorno abierto más acorde con plataformas virtuales externas al centro educativo.

- Poder conocer y analizar cómo aprenden los estudiantes de otros países (Finlandia y Chequia) al tener acceso a sus producciones audiovisuales compartidas.

- Aumentar la conciencia de globalidad, de pertenecer y tener influencia en el aprendizaje y en el desarrollo de proyectos que tienen lugar en otros centros y/o países.

- Aprender de otras culturas educativas y de otros modos de trabajar y entender el aprendizaje en los centros educativos.

- Aprender a desarrollar cooperativamente y colaborativamente un proyecto docente experimentando con plataformas digitales de acceso abierto.

- Compartir experiencias de aprendizaje pudiendo vislumbrar la multiculturalidad europea en las aulas.

\section{Implementación de DIYLab: fortalezas y retos}

En función de las dimensiones analizadas en los grupos de discusión finales, presentamos los resultados significativos sobre las fortalezas y retos del proyecto identificados por profesorado, alumnado y familias. 


\section{a) Aprendizaje con DIYLab}

Los estudiantes valoraron positivamente haber trabajado en grupos basados en sus intereses, tener más libertad en la toma de decisiones, poder ocupar otros espacios de la escuela fuera del aula y trabajar con ordenadores o dispositivos móviles de una forma más interactiva, autónoma y creativa.

El profesorado admitió que introducir la dimensión reflexiva en los procesos de enseñanza y aprendizaje (mediante las producciones audiovisuales de los estudiantes donde actuaban como reporteros y se entrevistaban entre ellos) fomentó que el alumnado tomara mayor consciencia de su propio aprendizaje. Esto les permitió poder autoreflexionar como docentes, aprendiendo a reconocer sus áreas de no saber, buscando otras estrategias para guiar al estudiante y además aprender con y al mismo tiempo que él.

Las familias valoraron positivamente el trabajar por intereses y colaborativamente dado que: 1) los estudiantes aprenden a trabajar en equipo, 2) descubren intereses compartidos que desconocían de sus compañeros, 3) aprenden a poner el interés común por encima de la amistad, 4) pueden compartir el conocimiento entre ellos más fácilmente, 5) aprenden a abrirse al grupo, a escuchar las propuestas de los compañeros y a respetar sus opiniones y formas de trabajar, y 6) aprenden a organizarse para llevar a cabo un trabajo conjunto.

\section{b) Relaciones: actitudes y roles}

Lo que más motivó a los estudiantes fue trabajar en actividades de forma transdisciplinar. El profesorado experimentó la evolución del rol docente como mediador que va negociando con el alumnado los puntos de intersección entre sus intereses y el currículo. A su vez, valoraba el trabajo por proyectos, el introducir más de un docente o investigador en el aula, y el uso de rúbricas de evaluación que permitían que el alumnado evaluara a sus compañeros y realizara su autoevaluación.

En cuanto a las familias, destacaron que trabajando de este modo algunos de los estudiantes se abren más a los demás, pierden miedos y ganan en seguridad, arriesgándose más en la toma de decisiones. A la vez que ellos desarrollan cierto compromiso y responsabilidad hacia el trabajo y sus compañeros, y que están más motivados en el aula.

\section{c) Competencia digital}

Los estudiantes valoraron positivamente poder trabajar con diferentes aplicaciones y servicios digitales. Por ejemplo, para hacer presentaciones en clase (destacan PowerPoint, Canva, Prezi y Google presentaciones) o trabajar en grupo de forma virtual (destacan Google Drive y aula virtual de la escuela). También, agradecieron poder usar diferentes dispositivos móviles (ordenadores portátiles, tabletas, cámaras digitales...) según las necesidades. Esto les permitió por ejemplo aprender a crear, grabar y editar vídeos, e ir mejorando todas estas acciones de forma progresiva.

El profesorado y las familias valoraron que los estudiantes fueran introduciendo las tecnologías digitales en su vida cotidiana hasta incorporarlas de manera muy natural como una herramienta de trabajo dentro y fuera del centro. Así, se abrían a nuevos 
modos de relacionarse con sus compañeros y de aprender. Todo esto permitió que se desdibujaran los saberes, generando espacios donde docentes o familias también aprenden de y con los estudiantes.

\section{d) Sostenibilidad del proyecto}

Según los estudiantes, el reto está en poder trasladar y extender la metodología desarrollada al resto de profesorado del centro consiguiendo una manera más divertida e interactiva de aprender. También reconocen, igual que los docentes, una necesidad de disponer de más recursos humanos, pues éstos no siempre pueden atenderlos a todos. Según los docentes, para poder garantizar cierta sostenibilidad del proyecto, es necesario repensar y pautar más las implicaciones del proyecto DIYLab. Finalmente, según las familias, este tipo de proyecto puede ser viable siempre y cuando se forme al profesorado y se prepare al alumnado.

\section{Implementación de DIYLab: debilidades o dificultades}

En función de las dimensiones analizadas en los grupos de discusión finales, presentamos los resultados significativos sobre las debilidades o dificultades del proyecto identificadas por profesorado, alumnado y familias.

\section{a) Aprendizaje con DIYLab}

Algunos docentes señalan que este tipo de proyecto no les parece del todo adecuado como práctica para el alumnado de primaria, debido a un problema de madurez o de un modo de hacer educativo. También, señalan que la flexibilidad e incertidumbre de la práctica docente requiere un proceso de adaptación que varía según las características y realidades de cada docente.

\section{b) Relaciones: actitudes y roles}

Algunos docentes reconocieron que no motiva a todos los estudiantes trabajar de esta forma, a la vez que para ellos hay contenidos importantes que este planteamiento no siempre permite introducir. También, destacan la necesidad de ir repensando progresivamente el rol del docente y de los estudiantes para poder participar e impulsar este proyecto. Los estudiantes muestran incomodidad cuando se tienen que tomar decisiones y/o asumir responsabilidades.

\section{c) Competencia digital}

Según el profesorado, el problema de madurez se vio acusado en relación a la búsqueda de información, ya que los estudiantes no sólo trabajaban en el aula virtual de la escuela (un espacio acotado) sino que se enfrentaban a una búsqueda abierta por Internet. Por su lado, las familias compartían una preocupación por un abuso innecesario del uso de las tecnologías digitales, dejando un poco al margen el tema de la gestión de las emociones. 


\section{d) Sostenibilidad del proyecto}

Según el profesorado, es necesario tener más en cuenta el currículo y no dejar tanta libertad de decisión a los estudiantes porque muchas veces se sienten perdidos. El cambio progresivo que requiere la sostenibilidad del proyecto se debería contemplar en la planificación y coordinación del profesorado.

\section{Discusión y conclusiones}

El proceso de cambio de la cultura docente que implica la consideración de la cultura DIY requiere un proceso participativo, colaborativo y democrático orientado a desarrollar el conocimiento práctico. No se puede promover desde una perspectiva jerárquica (de arriba abajo). La investigación-acción colaborativa y los distintos métodos de recogida de información se han revelado como los modos más adecuados para impulsar y documentar una transformación de la cultura docente del centro desde un proceso colaborativo.

El análisis de la implementación del proyecto DIYLab y, en particular, de las producciones audiovisuales creadas por alumnado de educación primaria, desvela una realidad educativa. Concretamente, la realidad de prestar atención a las distintas necesidades de los centros educativos de primaria que desean avanzar hacia una escuela centrada en los alumnos y el mundo en el que viven, y hacia una escuela que facilite el aprendizaje vivencial, con sentido y continuado. Seguidamente, destacamos las necesidades más significativas:

- Pensar las relaciones pedagógicas de modo más transversal para ir contemplando la capacidad de acción y el conocimiento de los estudiantes, en lugar de un solo "experto": el docente. Así, los saberes relativos se ponen en juego y representan una oportunidad para que las clases se conviertan en espacios y tiempos interesantes para todos los implicados. Esto requiere repensar la noción de currículo y de aprendizaje, y partir de los intereses y conocimientos de los estudiantes para transformarlos en contenidos o materia docente superando la rigidez del currículo (Meléndez, 2015, p. 51).

- Revisar el rol del docente para avanzar hacia el de ser creador de circunstancias para su propio aprendizaje y el aprendizaje del alumnado. En esta perspectiva de aprendizaje, el profesorado se convierte en guía, facilitador y diseñador de experiencias de aprendizaje centradas en el alumnado y el mundo que le rodea (Weimer, 2013). Esta revisión permite apostar por un rol del estudiante con conciencia de aprender a lo largo y ancho de la vida (Banks et al., 2007), evitando un aprendizaje o una enseñanza exclusivamente centrada en el docente, las áreas, las materias y los exámenes.

- Evitar la rigidez de la distribución de los contenidos en compartimentos estancados de horarios y materias como factor limitante, apostando por equipos interdisciplinares con proyectos en franjas de tiempo flexibles. Esto facilita generar más espacios para atender y dar respuesta a los intereses del estudiante favoreciendo la toma de decisiones sobre qué y cómo trabajar (Hernández-Hernández, 2006). 
- Prestar atención a los procesos emocionales que son parte del aprender, como la toma de decisiones o el generar un ambiente que propicie la autoconfianza de los estudiantes (Maturana, 1990). A la vez, favorecer un contacto más directo con la realidad del alumnado integrando la vida de los niños en el contexto de la escuela. Coincidimos con Thompson (2014) que los procesos emocionales pueden motivar comportamientos y decisiones. Por lo tanto, reducir la importancia de los procesos emocionales del aprendizaje puede tener consecuencias negativas para estudiantes y docentes (Edgington, 2016, p. 72).

- Trabajar la competencia digital de forma colaborativa para aprender. Se trata, en definitiva, de ir más allá de los aspectos puramente técnicos para llenarla de sentido pedagógico (Calvani, Fini, \& Ranieri, 2010, p. 161). Según Boyd (2014, p. 13), el hecho de que los jóvenes usen medios de comunicación social, no significa que todos sean experimentados por igual o que dispongan automáticamente de las habilidades para utilizarlos. Los jóvenes suelen sentirse cómodos usándolos, pero siguen transitando de manera incómoda entre dependencia e independencia. Esto se aprecia por ejemplo cuando reclaman pautas para realizar proyectos o se muestran incómodos cuando tienen que tomar decisiones y/o asumir responsabilidades.

Las limitaciones de este proyecto no se encuentran en los métodos utilizados, sino en el desafío que supone introducir en la cultura institucional unas perspectivas y unas prácticas de aprendizaje y colaboración desarrolladas en ambientes no formales sin sistemas de evaluación estandarizados. Actualmente (una vez acabado este proyecto europeo), los investigadores seguimos profundizando en el estudio de las producciones audiovisuales como modos de representación y transferencia de los aprendizajes. También, seguimos profundizando en la articulación de proyectos basados en la indagación que fomenten las seis características del aprendizaje DIY anteriormente señaladas (creatividad, colaboración, autorregulación, autoría, compartición y uso de tecnologías).

Referente a las aportaciones o consecuencias prácticas del trabajo realizado con la implementación de este proyecto DIYLab, destacaríamos qué hemos conseguido como más significativo:

- Fomentar una actitud proactiva en docentes y estudiantes en lo referente a la regulación de su propio aprendizaje.

- Introducir la visión transdisciplinar del conocimiento mediante proyectos basados en la indagación.

- Mostrar la capacidad del alumnado para informar de manera narrativa sobre sus trayectorias de aprendizaje.

- Evidenciar el dominio de los medios digitales y visuales para vincularlos a las narraciones.

- Mostrar la capacidad de colaboración entre docentes, estudiantes y/o investigadores para preparar un guion, estructurar y organizar ideas, y plasmarlas en productos audiovisuales.

- Evidenciar la mejora de las habilidades y destrezas relacionadas con las competencias digital, reflexiva, analítica, crítica e investigadora. 
- Promover un compartir el conocimiento y los aprendizajes desde una plataforma digital de acceso abierto y de manejo fácil (DIYLabHub).

- Fomentar la transdisciplinariedad, interculturalidad e intergeneracionalidad de los procesos de enseñanza y aprendizaje a través del DIYLabHub.

- En definitiva, ir transformando las prácticas de enseñanza y aprendizaje del alumnado de educación primaria.

A título de cierre, aportamos una valoración relevante de la directora de la Escola Virolai sobre qué ha implicado para su centro la implementación del proyecto DIYLab. Ella nos comentó que les ha implicado "avanzar hacia una escuela para aprender incorporando dinámicas de reflexión sobre y desde el aprendizaje del alumnado y del profesorado".

\section{Apoyos}

Comisión Europea. Lifelong Learning Programme; and the Education, Audiovisual and Culture Executive Agency. 543177-LLP-1-2013-1-ES-KA3MP. [Descargo de responsabilidad: este artículo refleja los puntos de vista de los autores y la Comisión no puede hacerse responsable de la información contenida].

Grupo de investigación ESBRINA-Subjetividades, visualidades y entornos educativos contemporáneos (2014SGR 00632).

Red de excelencia REUNI+D - Red Universitaria de Investigación e Innovación Educativa. Cambios Sociales y Retos para la Educación en la Era Digital. EDU201568718-REDT.

Programa de ayudas a la Formación del Profesorado Universitario del Ministerio de Educación, Cultura y Deporte, FPU13/03637.

\section{Referencias}

Ala-Mutka, K. (2009). Review of Learning in ICT-enabled Networks and Communities. European Commission. Institute for Prospective Technological Studies. Recuperado de https://goo.gl/g12EDL

Ashworth, H. (2016). Students' acquisition of a threshold concept in childhood and youth studies. Innovations in Education and Teaching International, 53(1), 94-103. doi: https://doi.org/10.1080/14703297.2014.1003953

Atkinson, D. (2011). Art, Equality and Learning: Pedagogies against the State. Rotterdam: Sense Publishers.

Banks, J. A., Au, K. H., Ball, A. F., Bell, P., Gordon, E. W., Gutiérrez, K., ... Zhou, M. (2007). Learning in and out of school in diverse environments. Life-long, life-wide, life-deep. The LIFE Center for Multicultural Education. Recuperado de https://goo. gl/Cu2R6J

Barbour, R., \& Kitzinger, J. (Ed.) (1999). Developing Focus Group Research: Politics, Theory and Practice. London, UK: Sage Publications Ltd. 
Bell, P., Tzou, C., Bricker, L., \& Baines, A. D. (2013). Learning in diversities of structures of social practice: Accounting for how, why and where people learn science. Human Development, 55(5-6), 269-284. doi: https://doi.org/10.1159/000345315

Bowen, G. A. (2009). Document Analysis as a Qualitative Research Method, Qualitative Research Journal, 9(2), 27-40. doi: https://doi.org/10.3316/QRJ0902027

Boyd, D. (2014). It's Complicated. The social lives of networked teens. New Haven, Connecticut, EE.UU: Yale University Press Book.

Buckingham, D. (2007). Beyond Technology: Children's Learning in the Age of Digital Culture. Cambridge, UK: Polity Press.

Calvani, A., Fini, A., \& Ranieri, M. (2010). Digital Competence. In K-12. Theoretical Models, Assessment Tools and Empirical Research. Anàlisi, 40, 157-171. doi: https:// doi.org/10.7238/a.v0i40.1151

Carey, B. (2014). How we learn: the surprising truth about when, where, and why it happens. New York, EE.UU: Random House.

Domingo-Coscollola, M., Arrazola-Carballo, J., \& Sancho-Gil, J. M. (2016). Do It Yourself in Education: Leadership for Learning across Physical and Virtual Borders. International Journal of Educational Leadership and Management, 4(1), 5-29. doi: https:// doi.org/10.17583/ijelm.2016.1842

Domingo-Coscollola, M., Sánchez-Valero, J. A., \& Sancho-Gil, J. M. (2014). Researching on and with Young People: Collaborating and Educating. Comunicar, 42(21), 157-164, doi: https://doi.org/10.3916/C42-2014-15

Dussel, I., \& Gutiérrez, D. (Comps.) (2006). Educar la mirada. Políticas y pedagogías de la imagen. Buenos Aires, Argentina: Manantial/FLACSO.

Edgington, U. (2016). Emotional labour in the classroom: Lessons from a history of the British education system. Journal of New Zealand Studies, 22, 72-86.

Erstad, O., Gile, O., Sefton-Green, J., \& Arnseth, H. C. (2016). Learning identities, education and community: young live in the cosmopolitancity. Cambridge, UK: Cambridge University Press.

Erstad, O., Kumpulainen, K., Mäkitalo, A., Schrøder, K. C., Pruulmann-Vengerfeldt, P., \& Jóhannsdóttir, T. (Eds.) (2016). Learning across contexts in the knowledge society. Rotterdam: Sense Publishers.

Escola Virolai (s.f.). Projecte Educatiu. Recuperado de http://www.virolai.com/projecte_ educatiu_ca.html

Fields, D., Vasudevan, V., \& Kafai, Y. B. (2015). The programmers' collective: fostering participatory culture by making music videos in a high school Scratch coding workshop. Interactive Learning Environments, 23(5), 613-633. doi: https://doi.org/10. 1080/10494820.2015.1065892

Furlong, J., \& Oancea, A. (Eds.). (2008). Assessing quality in applied and practice-based research in education: continuing the debate. London, UK: Routledge.

García-Galera, C., \& Valdivia, A. (2014). Prosumidores mediáticos. Cultura participativa de las audiencias y responsabilidad de los medios. Comunicar, 43(22), 10-13. doi: https://doi.org//10.3916/C43-2014-a2

García-Valcárcel, A., \& Basilotta, V. (2017). Aprendizaje basado en proyectos (ABP): evaluación desde la perspectiva de alumnos de Educación Primaria. Revista de Investigación Educativa, 35(1), 113-131. doi: http://dx.doi.org/10.6018/rie.35.1.246811 
Gutiérrez, A., \& Tyner, K. (2013). Educación para los medios, alfabetización mediática y competencia digital. Comunicar, 38(19), 31-39. doi: https://doi.org/10.3916/C382011-02-03

Hernández-Hernández, F. (2006). ¿Por qué decimos que estamos a favor de la educación si optamos por un camino que deseduca y excluye? En J. M. Sancho-Gil (Coord.), Tecnologías para transformar la educación (pp. 51-75). Madrid: AKAL/UNIA.

Hernández-Hernández, F. (Coord.). (2017) ¡Y luego dicen que la escuela pública no funciona! Investigar con jóvenes cómo transitan y aprenden dentro y fuera de los centros de Secundaria. Barcelona: Octaedro.

Hernández-Hernández, F., \& Domingo-Coscollola, M. (2017). Jóvenes que participan en una investigación para ser reconocidos. En F. Hernández-Hernández (Coord.), ¡Y luego dicen que la escuela pública no funciona! Investigar con jóvenes cómo transitan y aprenden dentro y fuera de los centros de Secundaria (pp. 241-284). Barcelona: Octaedro.

Ito, M., Gutiérrez, K., Livingstone, S., Penuel, B., Rhodes, J., Salen, K., ... Watkins, S. C. (2013). Connected Learning: An Agenda for Research and Design. Irvine, California, EE.UU: Digital Media and Learning Research Hub.

Jenkins, H., Purushotma, R., Weigel, M., Clinton, K., \& Robison, A. J. (2009). Confronting the Challenges of Participatory Culture. Media Education for the 21st Century. Cambridge, Massachusetts, EE.UU: MIT Press.

Jenson, J., Dahya, N., \& Fisher, S. (2014). Valuing production values: a "do it yourself" media production club. Learning, Media and Technology, 39(2), 215-228. doi: https:// doi.org/10.1080/17439884.2013

Kafai, Y. B., \& Peppler, K. A. (2011). Youth, Technology, and DIY: Developing Participatory Competencies in Creative Media Production. Review of Research in Education, 35, 89-119. doi: https://doi.org/10.3102/0091732x10383211

Kafai, Y. B., \& Peppler, K. A. (2014). Transparency Reconsidered: Creative, Critical and Connected Making with E-Textiles. En M. Ratto \& M. Boler (Eds.), DIYCitizenship. Critical Making and Social Media (pp. 179-188). Cambridge, Massachusetts, EE.UU: MIT Press.

Knobel, M., \& Lankshear, C. (Eds.). (2010). DIY Media: Creating, Sharing and Learning with New Technologies. New York, EE.UU: Peter Lang Publishing.

Livingstone, S., \& Sefton-Green, J. (2016). The Class: living and learning in the digital age. New York, EE.UU: New York University Press.

Maturana, H. (1990). Emociones y lenguaje en educación y política. Santiago de Chile, Chile: Ediciones Dolmen.

Meléndez, R, (2015). Educación - Currículo y Sociedad. Revista de Tecnología de Información y Comunicación en Educación, 9(2), 51-62.

Palaigeorgiou, G., \& Grammatikopoulou, A. (2016). Benefits, barriers and prerequisites for Web 2.0 learning activities in the classroom: The view of Greek pioneer teachers. Interactive Technology and Smart Education, 13(1), 2-18. doi: https://doi.org/10.1108/ ITSE-09-2015-0028

Peppler, K. (2013). STEAM-powered computing education: Using e-textiles to integrate the arts and STEM. IEEE Computer 46(9), 38-43. doi: https://doi.org/10.1109/ MC.2013.257 
Pérez-Escoda, A., Castro-Zubizarreta, A., \& Fandos, M. (2016). La competencia digital de la Generación Z: claves para su introducción curricular en la Educación Primaria. Comunicar, 49(24), 71-79. doi: https://doi.org/10.3916/C49-2016-07

Reason, P., \& Bradbury, H. (Eds.). (2001). Handbook of action research: Participative inquiry and practice. London, UK: Sage.

Richard, G. T., \& Kafai, Y. B. (2016). Blind Spots in Youth DIY Programming: Examining Diversity in Creators, Content, and Comments within the Scratch Online Community. In Proceedings of the 2016 CHI Conference on Human Factors in Computing Systems (pp. 1473-1485). New York, EE.UU: ACM.

Sharpe, R., Beethan, H., \& De Freitas, S. (2010). Rethinking Learning for a Digital Age: How Learners are Shaping Their Own Experiences. New York, EE.UU: Routledge.

Shirky, C. (2010). Cognitive Surplus: How Technology Makes Consumers into Collaborators. New York, EE.UU: Penguin.

Thomas, T., \& Brown, J. S. (2011). A New Culture of Learning: Cultivating the Imagination for a World of Constant Change. Lexington, Kentucky, EE.UU: Create Space.

Thompson, V. A. (2014). What intuitions are... and are not. Psychology of Learning and Motivation, 60, 35-75. doi: https://doi.org/10.1016/B978-0-12-800090-8.00002-0

Van Maanen, J. (2011). Tales of the field: on writing ethnography. Chicago, Illinois, EE.UU: University of Chicago Press.

Weimer, M. (2013). Learner-centered teaching: five key changes to practice. San Francisco, California, EE.UU: Jossey-Bass.

Zimmerman, B. J. (1989). Models of Self-Regulated Learning and Academic Achievement. In B. J. Zimmerman \& D. H. Schunk (Eds.), Self-Regulated Learning and Academic Achievement. Theory, Research, and Practice. New York, EE.UU: Springer-Verlag.

Fecha de recepción: 16 de septiembre de 2017

Fecha de revisión: 30 de septiembre de 2017

Fecha de aceptación: 13 de febrero de 2018 